\title{
Determination of three-dimensional distribution of electrical conductivity in the Earth's mantle from Swarm satellite data: Time-domain approach
}

\author{
Jakub Velímský \\ Department of Geophysics, Faculty of Mathematics and Physics, Charles University in Prague, Czech Republic
}

(Received January 11, 2013; Revised June 28, 2013; Accepted August 6, 2013; Online published November 22, 2013)

\begin{abstract}
One of the primary goals of the Swarm multisatellite mission is to determine the 3-D distribution of electrical conductivity in the Earth's mantle. This paper presents an inversion method based on direct integration of magnetic fields in the time domain, and using the adjoint solution for fast evaluation of data sensitivities to model perturbations. Two tests of the method are presented. The first one is using a 3-D checkerboard conductivity model and noise-free synthetic data. The second test is based on the closed-loop simulation of Swarm mission, including recovery of external and induced fields from simulated data along satellite tracks, and realistic noise estimates.
\end{abstract}

Key words: 3-D electromagnetic induction, 3-D inversion, mantle conductivity anomalies, time domain.

\section{Introduction}

The method of electromagnetic (EM) induction has long been a standard geophysical tool used to study the electrical conductivity of the Earth (Lahiri and Price, 1939; Banks, 1969). On a planetary scale, long-period data (in the period range from few hours to years) from permanent geomagnetic observatories have been traditionally used in 1-D inversions for depth-dependent mantle conductivity structure. Availability of new geomagnetic measurements from low-altitude satellites and from the expanding observatory network has also inspired development of fully threedimensional (3-D) techniques (Koyama et al., 2006; Kelbert et al., 2008, 2009; Shimizu et al., 2010; Tarits and Mandea, 2010; Kuvshinov and Semenov, 2012; Semenov and Kuvshinov, 2012). Thorough reviews of recent advancements in the area of global EM induction are presented by Kuvshinov $(2008,2012)$.

Most of these methods work in the frequency domain, for which the EM induction equation is solved at discrete time-harmonics. The time-domain approaches introduced by Hamano (2002) and Velímský and Martinec (2005) allow to model EM induction due to transient excitation. This paper presents the development of a 3-D time-domain inversion scheme tailored for the upcoming Swarm multisatellite mission as part of the Swarm Satellite Constellation Application and Research Facility (Olsen et al., 2013, SCARF). The algorithm uses the time-series of external field Gauss coefficients, and their induced counterparts, obtained by the comprehensive inversion (Sabaka et al., 2013, CI) of Swarm satellite and ground observatory data, and inverts them in terms of 3-D electrical conductivity structure in the Earth's mantle.

Copyright (C) The Society of Geomagnetism and Earth, Planetary and Space Sciences (SGEPSS); The Seismological Society of Japan; The Volcanological Society of Japan; The Geodetic Society of Japan; The Japanese Society for Planetary Sciences; TERRAPUB.

doi:10.5047/eps.2013.08.001
The key features of the algorithm are described in Section 2. The results of two basic tests of the method: a noisefree checkerboard test demonstrating the spatial resolution under ideal conditions, and a closed-loop test allowing for direct comparison with the complementary frequencydomain chain (Püthe and Kuvshinov, 2013, 3FDI), are presented in Section 3. In the last section, the results, and outlooks for further development of the method are summarized.

Electrical resistivity $\rho$ is used throughout Section 2 to allow simple notation in the $\boldsymbol{B}$-field formulation, and to preserve compatibility between the manuscript and the actual software implementation. Electrical conductivity $\sigma=1 / \rho$ is preferred in Section 3, discussions, and plots for easy comparison with the accompanying paper (Püthe and $\mathrm{Ku}-$ vshinov, 2013).

\section{Inverse Modelling in the Time Domain \\ 2.1 Forward problem}

A detailed description of the forward solver used in this work is given by Velímský and Martinec (2005). Here we give only a brief overview.

The magnetic field $\boldsymbol{B}(\boldsymbol{r} ; t)$ inside a conductive, nonmagnetic, solid spherical Earth of radius $a$ with threedimensional, isotropic distribution of electrical resistivity $\rho(\boldsymbol{r})>0$, and under the magneto-quasistatic approximation $(\sigma \gg \omega \epsilon)$, is subject to the induction equation,

$$
\nabla \times[\rho(\boldsymbol{r}) \nabla \times \boldsymbol{B}(\boldsymbol{r} ; t)]+\mu_{0} \frac{\partial \boldsymbol{B}(\boldsymbol{r} ; t)}{\partial t}=0 .
$$

Here $\boldsymbol{r}=(r, \Omega)=(r, \vartheta, \varphi)$ is the position vector described by radius, colatitude, and longitude in spherical coordinates, $t$ is time, and $\mu_{0}$ is the magnetic permeability, assumed to be that of non-magnetic geomaterial in the scope of global EM induction problem. The magnetic field in the insulating atmosphere above the conductive sphere, but below the region of magnetospheric electric currents (which 
are present at radial distances greater than some radius $b$ ) is described by scalar magnetic potential $U(\boldsymbol{r} ; t)$,

$$
\boldsymbol{B}(\boldsymbol{r} ; t)=-\nabla U(\boldsymbol{r} ; t) .
$$

The potential satisfies the Laplace equation,

$$
\Delta U(\boldsymbol{r} ; t)=0, \quad a \leq r \leq b,
$$

and therefore can be written in terms of infinite series of real, fully normalized spherical harmonic functions $Y_{j m}(\Omega)$,

$$
\begin{aligned}
U(\boldsymbol{r} ; t)=a \sum_{j=1}^{\infty} \sum_{m=-j}^{j}[ & G_{j m}^{(\mathrm{e})}(t)\left(\frac{r}{a}\right)^{j} \\
& \left.+G_{j m}^{(\mathrm{i})}(t)\left(\frac{a}{r}\right)^{j+1}\right] Y_{j m}(\Omega) .
\end{aligned}
$$

The setup of the problem doesn't take into account ionospheric electric currents flowing above the Earth's surface, but below the satellite orbit, and their induced counterparts in the Earth (Kuvshinov, 2008, and references therein). These signals must be modelled separately, and subtracted from the magnetic field in the preprocessing, making use of linearity of the induction equation (1). This approach is indeed applied in the CI processing of satellite data (Sabaka et al., 2013), which employs also the ground observatory data to resolve the ionospheric currents from the induced currents.

The time series of coefficients $G_{j m}^{(\mathrm{e})}(t)$, and $G_{j m}^{(\mathrm{i})}(t)$ describe the spatio-temporal evolution of the primary magnetic field of the electric currents in the magnetosphere, and the secondary electric currents induced in the conductive Earth, respectively. They are related to the traditional set of Gauss coeffcients $g_{j m}, h_{j m}, q_{j m}, s_{j m}$ in Schmidt's seminormalization (Langel, 1987) by equations,

$$
\begin{aligned}
& G_{j m}^{(\mathrm{i})}=\sqrt{\frac{4 \pi}{2 j+1}} \begin{cases}g_{j m} & m=0, \\
(-1)^{m} g_{j m} & m>0, \\
h_{j|m|} & m<0,\end{cases} \\
& G_{j m}^{(\mathrm{e})}=\sqrt{\frac{4 \pi}{2 j+1}} \begin{cases}q_{j m} & m=0, \\
(-1)^{m} q_{j m} & m>0, \\
s_{j|m|} & m<0 .\end{cases}
\end{aligned}
$$

The series of coefficients, and therefore the summation in Eq. (4) is truncated at a finite degree $j_{\max }$. Assuming continutity of magnetic field $\boldsymbol{B}$ across the Earth's surface $r=a$, and expressing $\boldsymbol{B}$ in the base of vector spherical harmonic functions combined with finite elements in the radial direction, the Eqs. (1)-(2) can be discretized and coupled. Given the resistivity $\rho(\boldsymbol{r})$, and the source model $G_{j m}^{(\mathrm{e})}(t)$, the forward solution is integrated from the initial condition,

$$
\boldsymbol{B}(\boldsymbol{r} ; 0)=\mathbf{0}
$$

to provide a time series of induced coefficients $G_{j m}^{(\mathrm{i})}(t)$.

The original approach to the integration introduced in Velímský and Martinec (2005) was based on a semi-implicit scheme that treated implicitly the dominant effect of 1-D resistivity profile for stability, while the effects of lateral variations were taken from the previous time step. However, this approach yields large inaccuracies in the evaluation of data sensitivities based on the adjoint problem solution, as described in the next section. Therefore, a new version of the program was developed (Kuvshinov et al., 2010), based on an unconditionally stable, second-order accurate Crank-Nicolson integration scheme (Press et al., 1992, chapter 19). Such approach leads to a banded linear system of equations; however, the width of the band can become large since it depends on $j_{\max }^{2}$. Factorization of a large, wide-banded linear system, and its repeated solutions at each time step are CPU-intensive. They were therefore paralellized on a distributed-memory architecture, using the ScaLAPACK and MPI libraries (Blackford et al., 1997). The matrix structure invites paralellization by layers, where each computational node stores the matrix and solution for at least 4 adjacent layers. Therefore, for optimal efficiency, the total number of layers in the forward modelling should be divisible by 4 .

\subsection{Inverse problem}

In order to formulate the inverse problem, the resistivity distribution in the Earth is first described by a finite number of model parameters. Spherical harmonic expansion in $K$ discrete layers is used, following similar setup by Kelbert $e t$ al. (2008) and Püthe and Kuvshinov (2013),

$$
\log _{10} \rho(\boldsymbol{r})=\sum_{k=1}^{K} \sum_{j=0}^{j_{\max }^{\rho}} \sum_{m=-j}^{j} \rho_{j m}^{k} \xi_{k}(r) Y_{j m}(\Omega)
$$

where $\xi_{k}(r)=1$ in the $k$-th layer, $r_{k} \leq r \leq r_{k+1}$, and is zero otherwise. Although this parameterization has no physical justification based on the likely geological processes in the mantle, detection of small-scale conductivity heterogeneities, such as related to the subducted crustal slabs in the mantle, is beyond the expected resolution of the Swarm dataset. Moreover, since the input data to the inverse problem are provided in the form of spherical harmonics, it is natural to choose the same basis also for the conductivity model. The coefficients $\rho_{j m}^{k}$ are arranged into the model vector $\boldsymbol{m}$ of dimension $M$. Not necessarily all coefficients are included in $\boldsymbol{m}$, and $M \leq K\left(j_{\max }^{\rho}+1\right)^{2}$. At the start of the inversion process, selected coefficients can be set to apriori values, and excluded from $\boldsymbol{m}$. This allows for a flexible setup of the inverse problem, such as combination of layers with different lateral resolution, and uniform layers.

To solve the inverse problem, one seeks a model $\tilde{\boldsymbol{m}}^{\lambda}$ that minimizes the penalty function

$$
F(\boldsymbol{m} ; \lambda)=\chi^{2}(\boldsymbol{m})+\lambda R^{2}(\boldsymbol{m}),
$$

where $\chi^{2}(\boldsymbol{m})$ is the data misfit, $\lambda$ is the regularization parameter, and $R^{2}(\boldsymbol{m})$ is the regularization term described in Subsection 2.3.

The data misfit $\chi^{2}(\boldsymbol{m})$ measures the difference between the observed data, and data predicted by forward modelling for given model $\boldsymbol{m}$. We define

$$
\chi^{2}(\boldsymbol{m})=\sum_{j m} \frac{2 j+1}{8 \pi\left(t_{1}-t_{0}\right)}
$$




$$
\begin{aligned}
& \times \int_{t_{0}}^{t_{1}}\left[j\left(\frac{G_{j m}^{(\mathrm{e})}(\boldsymbol{m} ; t)-G_{j m}^{(\mathrm{e}, \mathrm{obs})}(t)}{\delta_{j m}^{(\mathrm{e})}(t)}\right)^{2}\right. \\
& \left.+(j+1)\left(\frac{G_{j m}^{(\mathrm{i})}(\boldsymbol{m} ; t)-G_{j m}^{(\mathrm{i}, \mathrm{obs})}(t)}{\delta_{j m}^{(\mathrm{i})}(t)}\right)^{2}\right] \mathrm{d} t .
\end{aligned}
$$

Symbols $G_{j m}^{(\mathrm{e}, \mathrm{obs})}(t)$, and $G_{j m}^{(\mathrm{i}, \mathrm{obs})}(t)$ denote the time series of external and internal field coefficients, provided in time interval $\left(t_{0}, t_{1}\right)$ with their respective error estimates $\delta_{j m}^{(\mathrm{e})}(t)$, $\delta_{j m}^{(\mathrm{i})}(t)$. They are obtained from the satellite data by the process of comprehensive inversion (Sabaka et al., 2013), which involves separation of fields of core, lithospheric, and magnetospheric origin, and their induced counterparts. Since this is basically a least-square fit of spherical harmonic coefficients into data along satellite tracks and on surface, the CI is capable of providing not only coefficient errors, but even full covariance matrices, including covariances between external and internal field coefficients.

The summation limits in Eq. (10) are the same as in Eq. (4). If a particular coefficient is not provided by the dataset, we set formally $1 / \delta_{j m}^{(\mathrm{e})}=0$ or $1 / \delta_{j m}^{(\mathrm{i})}=0$. The $G_{j m}^{(\mathrm{i})}(\boldsymbol{m} ; t)$ coefficients are predicted by the forward solver for each particular model $\boldsymbol{m}$. As for the external field which is required as the boundary condition for the forward solver, the obvious choice is setting $G_{j m}^{(\mathrm{e})}(\boldsymbol{m} ; t)=G_{j m}^{(\mathrm{e}, \mathrm{obs})}(t)$, independent of $\boldsymbol{m}$, which also cancels the first part of integral in Eq. (10). This means that we assume perfect knowledge of the source field, and discard any information about its uncertainty provided by errors $\delta_{j m}^{(\mathrm{e})}(t)$. The time interval $\left(t_{0}, t_{1}\right)$ can span the entire duration of the mission or only selected subset. However, the initial time $t_{0}$ at which the evaluation of misfit starts should be chosen at least few days later than the zero time of the initial condition (7) of the forward problem. In this way, the transient (switch-on) effect is minimized (Velímský and Martinec, 2005; Velímský et al., 2006).

Two additional properties of definition (10) are worth mentioning. Firstly, in the (however unlikely) case of error estimates independent of spherical harmonic degree and order, $\delta_{j m}^{(\mathrm{e})}(t)=\delta_{j m}^{(\mathrm{i})}(t)=\delta B(t)$, Eqs. (2) and (4) lead to

$$
\begin{aligned}
& \chi^{2}(\boldsymbol{m})=\frac{1}{2 S\left(t_{1}-t_{0}\right)} \\
& \times \int_{S} \int_{t_{0}}^{t_{1}}\left[\frac{\boldsymbol{B}(\boldsymbol{m} ; a, \Omega ; t)-\boldsymbol{B}^{(\mathrm{obs})}(a, \Omega ; t)}{\delta B(t)}\right]^{2} \mathrm{~d} t \mathrm{~d} S,
\end{aligned}
$$

where $S=4 \pi a^{2}$ is the surface of the spherical Earth. Therefore, the data misfit corresponds to a dimensionless, weighted $L^{2}$ norm of differences between the predicted and observed magnetic fields over the Earth's surface and time. The presence of $j,(j+1)$, and $(2 j+1)$ factors in Eq. (10) is thus justified. Secondly, a straightforward generalization of definition (10) that would take into account entire covariance matrices is possible. Use of the time-dependent covariance matrices, or, at least, their diagonal parts $\delta_{j m}^{(\mathrm{i})}(t)^{-2}$, allows selective downweighting of coefficients poorly constrained by the CI, i.e., due to temporary data gaps at individual satellites, or a particulary disadvantageous geometry of the satellite constellation. However, allowing for uncertainties in the external field would require its coefficients to be incorporated in the model vector $\boldsymbol{m}$, and then recovered by the inversion jointly with the conductivity model.

Returning back to the problem of minimization of penalty function $F(\boldsymbol{m} ; \lambda)$, several effective multidimensional algorithms, such as conjugate gradients or quasi-Newton method (Press et al., 1992, chapters 10.6-7), are based on the computation of the gradient of the function $F(\boldsymbol{m} ; \lambda)$ in the space of model parameters,

$$
\nabla_{\boldsymbol{m}} F(\boldsymbol{m} ; \lambda)=\nabla_{\boldsymbol{m}} \chi^{2}(\boldsymbol{m})+\lambda \nabla_{\boldsymbol{m}} R^{2}(\boldsymbol{m}) .
$$

Effective evaluation of the first term is not trivial. The straightforward approach would be to solve $2 M+1$ forward problems for small perturbations of the model $\boldsymbol{m}$ into each direction in the model space. Then, by numerical differentiation, we could obtain an approximation of $\nabla_{\boldsymbol{m}} \chi^{2}$.

Here we use a different approach based on the adjoint method (McGillivray et al., 1994; Dorn et al., 1999; Fichtner, 2011). Direct differentiation of (10) yields

$$
\begin{aligned}
\nabla_{\boldsymbol{m}} \chi^{2}= & \sum_{j m} \frac{(2 j+1)(j+1)}{4 \pi\left(t_{1}-t_{0}\right)} \\
& \times \int_{t_{0}}^{t_{1}} \frac{G_{j m}^{(\mathrm{i})}-G_{j m}^{(\mathrm{i}, \mathrm{obs})}}{\delta_{j m}^{(\mathrm{i})}(t)^{2}} \nabla_{\boldsymbol{m}} G_{j m}^{(\mathrm{i})} \mathrm{d} t .
\end{aligned}
$$

The adjoint method allows us to evaluate $\nabla_{\boldsymbol{m}} \chi^{2}$ without explicit knowledge of $\nabla_{\boldsymbol{m}} G_{j m}^{(\mathrm{i})}$. It reduces the computational burden to the solution of one forward and one adjoint problem. Following a similar derivation as for the 2-D axisymmetric case (Martinec and Velímský, 2009), we define the adjoint magnetic field $\hat{\boldsymbol{B}}(\boldsymbol{r} ; \hat{t})$, and magnetic potential $\hat{U}(\boldsymbol{r} ; \hat{t})$, as solution of the adjoint problem

$$
\begin{aligned}
\nabla \times[\rho(\boldsymbol{r}) \nabla \times \hat{\boldsymbol{B}}(\boldsymbol{r} ; \hat{t})] & \\
+\mu_{0} \frac{\partial \hat{\boldsymbol{B}}(\boldsymbol{r} ; \hat{t})}{\partial \hat{t}} & =0, \quad r \leq a, \\
\hat{\boldsymbol{B}}(\boldsymbol{r} ; \hat{t}) & =-\nabla \hat{U}(\boldsymbol{r} ; \hat{t}), \quad r=a, \\
\Delta \hat{U}(\boldsymbol{r} ; \hat{t}) & =0, \quad r \geq a .
\end{aligned}
$$

The adjoint time, $\hat{t}=t_{1}-t$, runs in the opposite direction to normal time, starting from the end of the dataset. The adjoint external field is given by the residua of the forward problem,

$$
\begin{aligned}
\hat{G}_{j m}^{(\mathrm{e})}(\hat{t})= & -\frac{j+1}{t_{1}-t_{0}} \\
& \times \int_{\max \left(t_{0}, t_{1}-\hat{t}\right)}^{t_{1}} \frac{G_{j m}^{(\mathrm{i})}(\boldsymbol{m} ; \tau)-G_{j m}^{(\mathrm{i}, \mathrm{obs})}(\tau)}{\delta_{j m}^{(\mathrm{i})}(\tau)^{2}} \mathrm{~d} \tau,
\end{aligned}
$$

and the initial condition for the adjoint field is

$$
\hat{\boldsymbol{B}}(\boldsymbol{r} ; \hat{t}=0)=\mathbf{0} .
$$

Note that the system of Eqs. (14)-(16) is equivalent to the forward problem (1)-(3), and can be solved with the identical numerical technique. Also, Eq. (17) yields $\hat{G}_{j m}^{(\mathrm{e})}(0)=0$, 
i.e., the adjoint source is compatible with the initial condition (18).

Application of the operator $\nabla_{\boldsymbol{m}}$ to Eq. (1), multiplication by $\hat{\boldsymbol{B}}$, integration over time interval $\left(t_{0}, t_{1}\right)$ and volume of the sphere $G$, and finally, use of the Gauss theorem and integration per parts, yields after some algebra,

$$
\begin{aligned}
\nabla_{\boldsymbol{m}} \chi^{2}(\boldsymbol{m})= & \frac{1}{4 \pi a^{3} \mu_{0}} \\
& \times \int_{t_{0}}^{t_{1}} \int_{G} \nabla_{\boldsymbol{m}} \rho(\boldsymbol{m} ; \boldsymbol{r})[\nabla \times \boldsymbol{B}(\boldsymbol{m} ; \boldsymbol{r} ; t)] \\
& \cdot[\nabla \times \hat{\boldsymbol{B}}(\boldsymbol{m} ; \boldsymbol{r} ; \hat{t})] \mathrm{d} V \mathrm{~d} t
\end{aligned}
$$

Differentiating the resistivity with respect to any parameter $\rho_{j m}^{k}$ is trivial,

$$
\frac{\partial}{\partial \rho_{j m}^{k}} \rho(\boldsymbol{m} ; \boldsymbol{r})=\ln 10 \rho(\boldsymbol{m} ; \boldsymbol{r}) \xi_{k}(r) Y_{j m}(\Omega) .
$$

Integration of (19) is therefore straightforward with only one caveat. In order to know the adjoint solution $\hat{\boldsymbol{B}}$ at time $\hat{t}$, Eq. (17) requires that the forward solution is known for all times $t \geq t_{1}-\hat{t}$. The forward and adjoint problems cannot be solved concurrently, the forward problem must be solved first, the entire solution $\boldsymbol{B}(\boldsymbol{r} ; t)$ stored, and then the time integration of Eq. (19) is performed together with the integration of the adjoint problem. The large memory requirements are handled by parallel distribution to the computational nodes, using the layer structure already established for the forward solver to minimize data transfers between nodes.

\subsection{Regularization}

The purpose of the regularization term is to control overfitting of data by unrealistic, spurious oscillations of the resistivity model. Two regularizations were implemented, constraining the first and second derivative, respectively,

$$
\begin{aligned}
& R_{1}^{2}(\boldsymbol{m})=\frac{1}{a} \int_{G}\left|\nabla \log _{10} \rho(\boldsymbol{m} ; \boldsymbol{r})\right|^{2} \mathrm{~d} V, \\
& R_{2}^{2}(\boldsymbol{m})=a \int_{G}\left|\Delta \log _{10} \rho(\boldsymbol{m} ; \boldsymbol{r})\right|^{2} \mathrm{~d} V .
\end{aligned}
$$

Because the forward solver represents resistivity on a grid, the spatial derivatives in the regularizations (21)-(22) are evaluated numerically using a simple 3-point finitedifference stencil (Fornberg, 1996).

The trade-off between the data misfit $\chi^{2}(\boldsymbol{m})$, and the regularization $R^{2}(\boldsymbol{m})$ in Eq. (9) is governed by the choice of the regularization parameter $\lambda$. The optimal value of $\lambda$ is selected by an L-curve analysis (Hansen, 1992). A local minimum $\tilde{\boldsymbol{m}}^{\lambda}$ of the penalty function $F(\lambda ; \boldsymbol{m})$ is found for several values of $\lambda$. Then, the data misfit $\chi^{2}\left(\tilde{\boldsymbol{m}}^{\lambda}\right)$ is plotted versus regularization $R^{2}\left(\tilde{\boldsymbol{m}}^{\lambda}\right)$, and the maximum inflection point $\tilde{\lambda}$ of the curve is found visually, using refined sampling of $\lambda$ in its vicinity, as necessary. The corresponding model $\tilde{\boldsymbol{m}}^{\tilde{\lambda}}$ then represents the solution of the regularized inverse problem.

\subsection{Quasi-Newton minimization}

Our implementation of quasi-Newton minimization closely follows the method described in Press et al. (1992, section 10.7). For a given $\lambda$, and starting model $\boldsymbol{m}_{0}^{\lambda}$, the algorithm gradually builds an approximation of the inverse Hessian from the values of $F\left(\boldsymbol{m}_{i}^{\lambda}\right)$ and $\nabla_{\boldsymbol{m}} F\left(\boldsymbol{m}_{i}^{\lambda}\right)$ using the Broyden-Fletcher-Goldfarb-Shanno formula. Line minimization along a given direction uses Brent's method to proceed to the next iteration point $\boldsymbol{m}_{i+1}^{\lambda}$. The iteration terminates when the difference of two successive models $\boldsymbol{m}_{i+1}^{\lambda}-\boldsymbol{m}_{i}^{\lambda}$, or the gradient $\nabla \boldsymbol{m} F\left(\boldsymbol{m}_{i}^{\lambda}\right)$ drops below a specified tolerance, or after a maximum number of iterations is reached. Experiments have shown that faster convergence is achieved when the initial model $\boldsymbol{m}_{0}^{\lambda}$ is chosen as the optimal result $\tilde{\boldsymbol{m}}^{\lambda^{\prime}}$ from a run with stronger regularization, $\lambda^{\prime}>\lambda$. Therefore, runs for individual values of $\lambda$ are chained in descending order.

\section{Results \\ 3.1 Checkerboard test}

In this section we present the results of two tests of the time-domain 3-D spherical conductivity inversion method. The purpose of the first test is to determine the resolution of the method under idealized conditions with no source of errors. The target 3-D conductivity model, as shown in the right panel of Fig. 1, is based on the 1-D conductivity profile of Kuvshinov and Olsen (2006), resampled to $200 \mathrm{~km}$ layers spanning the entire crust and mantle. Using the expansion (8), the 1-D model thus defines the coefficients $\rho_{00}^{k}$. Over the 1-D background a 3-D checkerboard-like structure is superimposed (Kelbert et al., 2008). The coefficients $\rho_{32}^{k}$ are set to \pm 1.0 with the sign alternating between layers. The scaling is such that the lateral contrast in each layer is about 0.8 orders of magnitude. All other coefficients describing the model are zero. At the top of the conductivity model, an empirical surface conductance map scaled to a common thickness of $10 \mathrm{~km}$ is imposed. It is based on bathymetry, topography, and electrical conductivity of seawater, crystalline rocks, and sediments, and assembled using the procedure described by Everett et al. (2003). The electrical conductivity of the core is assumed to be $10^{5} \mathrm{~S} \cdot \mathrm{m}^{-1}$.

The target conductivity model is excited by an external magnetic field represented by spherical harmonic coefficients for orders $j=1,2,3$, and degrees $m=-1,0,1$. The length of time series is 4 years with uniform $1.5 \mathrm{hr}$ sampling interval. The induced field coefficients are evaluated by the forward solver up to degree and order 5. Homogeneous error $\delta_{j m}^{(\mathrm{i})}(t)=1 \mathrm{nT}$ is assumed. These settings represent a rather optimistic expectation of the spatio-temporal resolution of magnetospheric field and their induced counterparts by the Swarm mission (Sabaka et al., 2013).

The time series is then inverted in terms of 3-D conductivity distribution. Because no noise is present, regularization is switched off by setting $\lambda=0$. The surface conductance map is not recovered by the inversion, but rather overlaid over each conductivity model in the process of inversion. The recovered model is shown in the left panel of Fig. 1. This result clearly demonstrates the resolution of the inversion with realistic spatio-temporal resolution of simulated Swarm data. In the depth range 400-1000 km the 3-D 

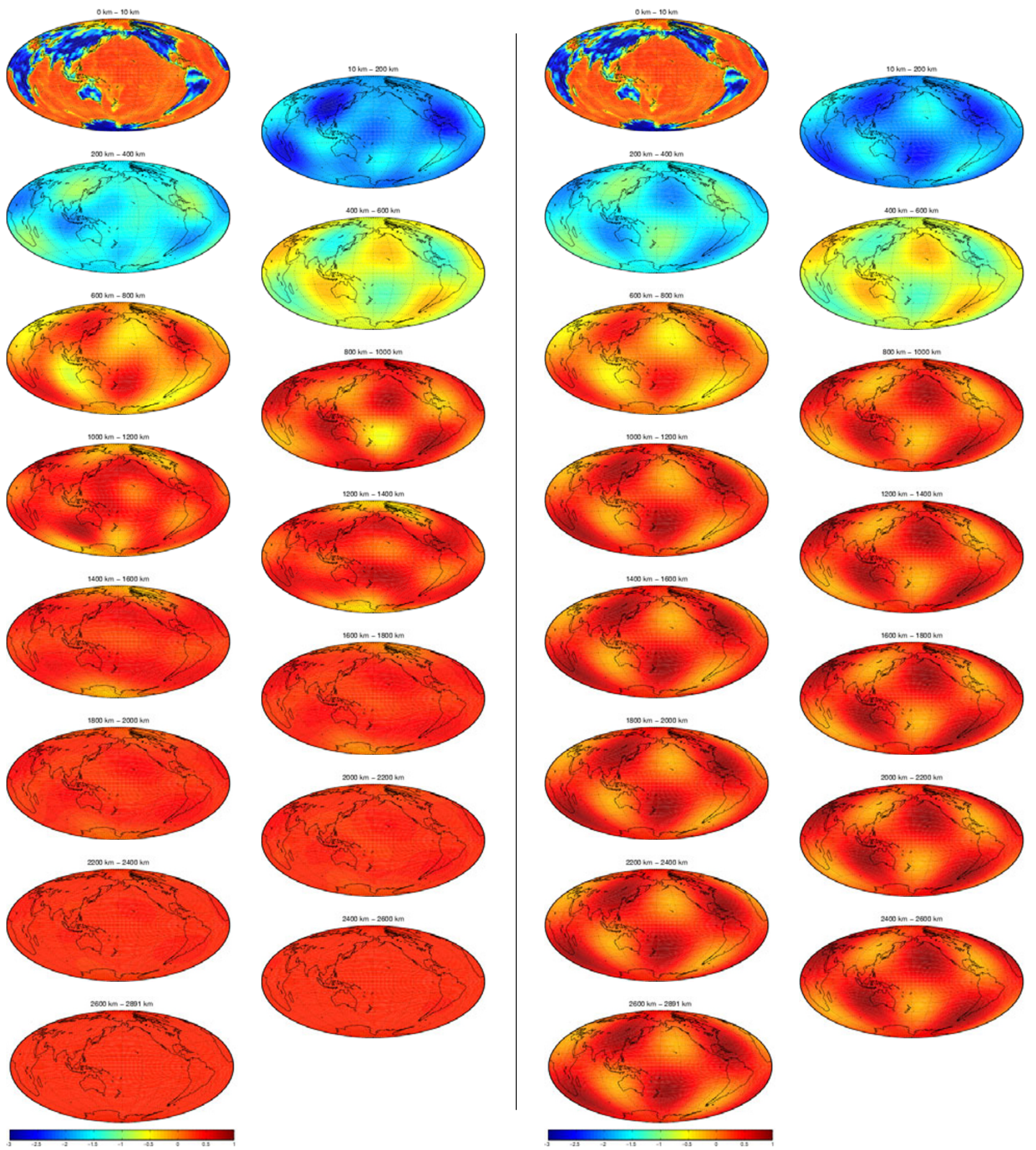

Fig. 1. Checkerboard test: Recovered model (left panel) compared to the target model (right panel). Color cross-sections through individual layers are staggered from top to bottom. Color scale corresponds to $\log _{10}\left(\sigma\right.$ in S.m $\left.{ }^{-1}\right)$.

structure is well-resolved. In the uppermost mantle, here represented by depth range $10-400 \mathrm{~km}$, the conductive and resistive heterogeneities are well resolved below the resistive continents. However, large difference between recovered and target model occurs below the oceans, especially the Pacific, a result of strong attenuation of the signal by highly conductive seawater. Below $1000 \mathrm{~km}$, the method is unable to resolve lateral variations of mantle conductivity, and only the 1-D average conductivity is recovered. Considerably longer time series would be needed for that task (Velímský and Finlay, 2011).

\subsection{Closed-loop test}

The second test case uses a target conductivity model that has been designed to test various elements of the SCARF (Olsen et al., 2013), in particular the CI (Sabaka et al., 2013), the 3FDI (Püthe and Kuvshinov, 2013), and the 3TDI (this paper).

From top to bottom, the model consists of the surface conductance map scaled to common thickness of $10 \mathrm{~km}$, a $390 \mathrm{~km}$ thick resistive layer $\left(0.004 \mathrm{~S} \cdot \mathrm{m}^{-1}\right)$ with three smallscale conductors embedded in it $\left(0.04 \mathrm{~S} \cdot \mathrm{m}^{-1}\right)$, a $300 \mathrm{~km}$ layer of conductivity of $0.04 \mathrm{~S} \cdot \mathrm{m}^{-1}$ with a large heterogene- 

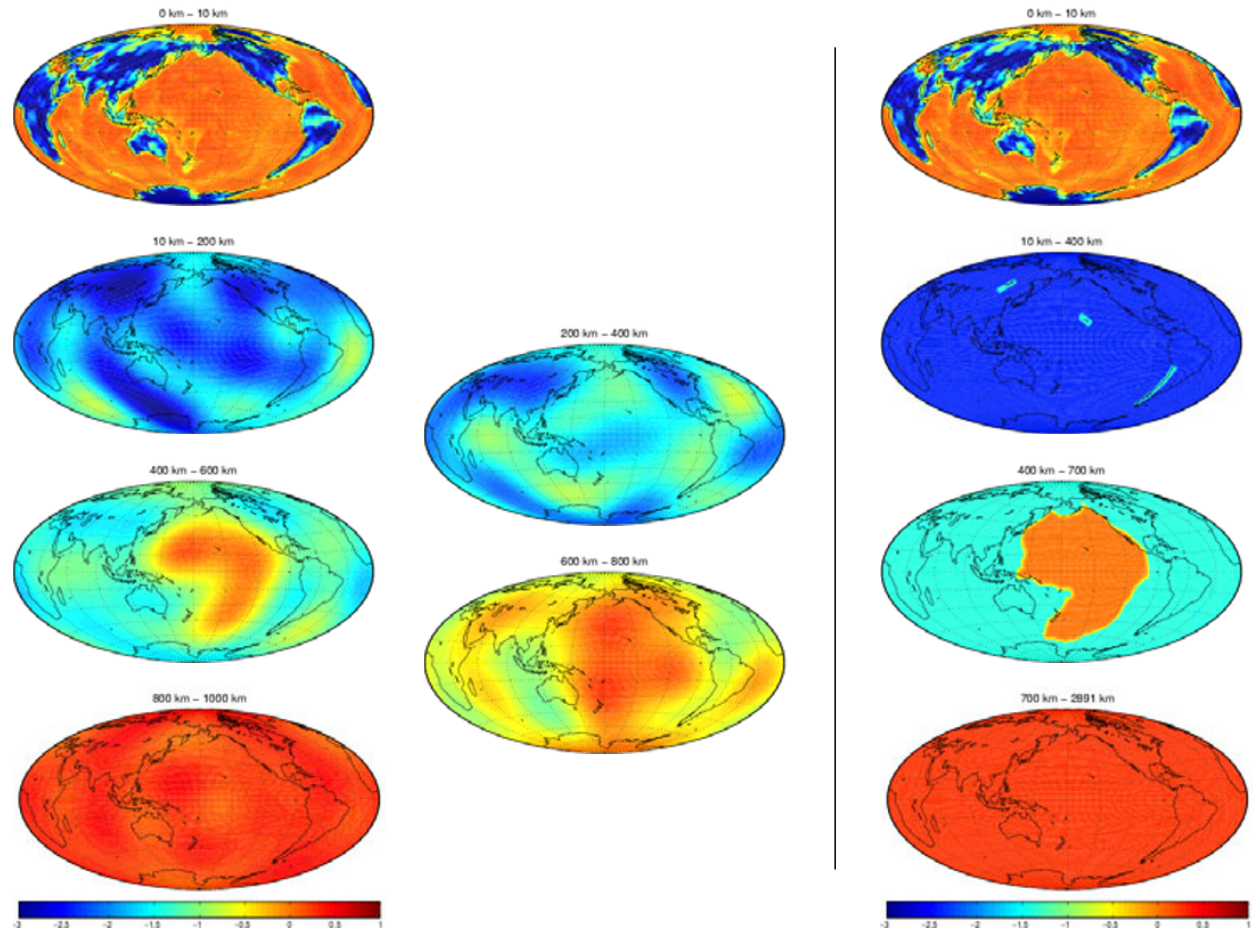

Fig. 2. Full closed-loop test: Recovered model (left panel) compared to the target model (right panel). Color cross-sections through individual layers are staggered from top to bottom. Color scale corresponds $\log _{10}\left(\sigma\right.$ in S. $\left.\mathrm{m}^{-1}\right)$, and is identical with the colorscale used in Fig. 3 in the companion 3FDI paper.

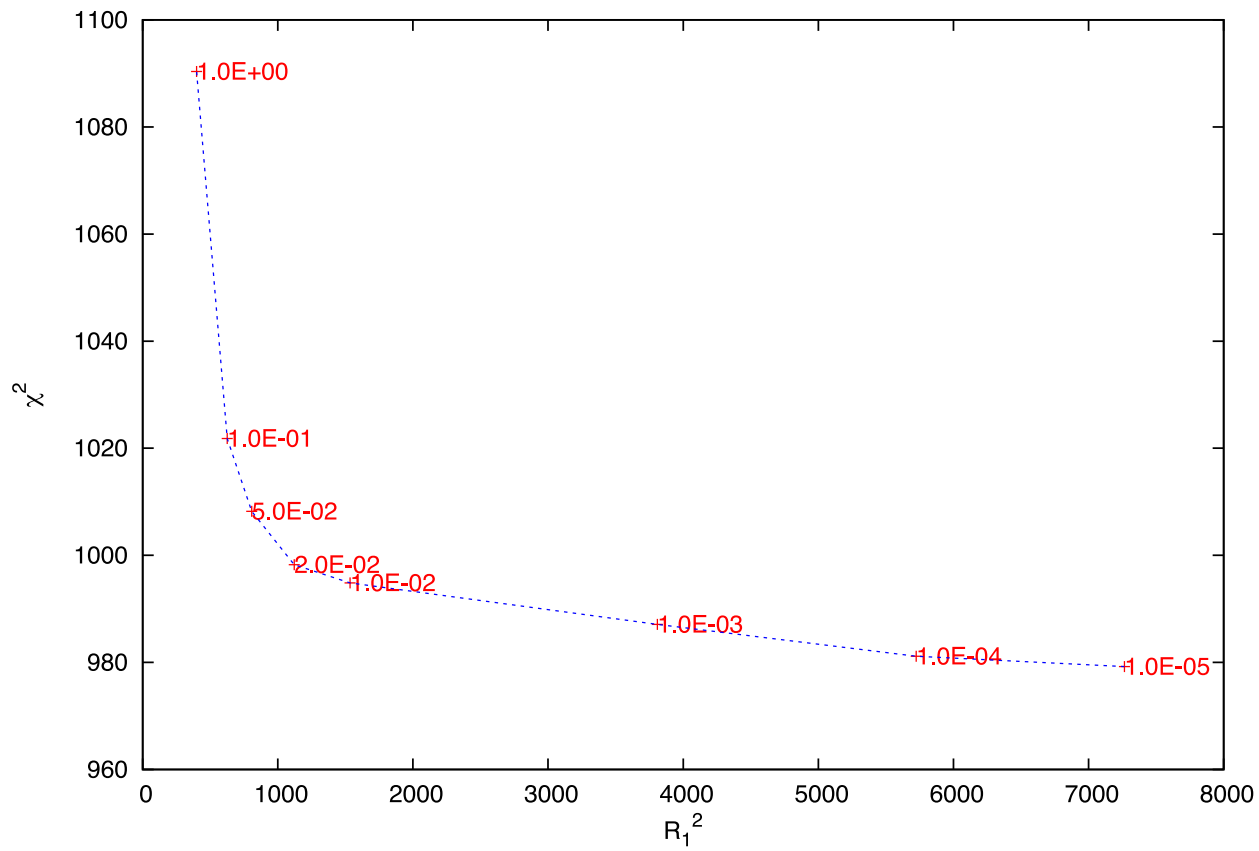

Fig. 3. L-curve used showing the data misfit $\chi^{2}(\boldsymbol{m})$ against the regularization term $R_{1}^{2}(\boldsymbol{m})$ for different values of parameter $\lambda$ (red numbers) in the closed-loop test.

ity of $1 \mathrm{~S} \cdot \mathrm{m}^{-1}$ approximately in the shape of the Pacific plate, a uniform conductor of $2 \mathrm{~S} \cdot \mathrm{m}^{-1}$ down to the coremantle boundary, and highly conductive core $\left(10^{5} \mathrm{~S} \cdot \mathrm{m}^{-1}\right)$. The model is shown in the right panel of Fig. 2.

An external field model up to degree 3 and order 1 (in the geomagnetic coordinate system), and with $1 \mathrm{hr}$ sampling interval was derived by analysis of 4.5 years of ground observatory hourly means from July 1998 to December 2002
(Olsen et al., 2005). Induced field coefficients for the target model were computed up to degree and order 15, using a frequency-domain integral equation solver (Pankratov et al., 1995). Details of the method, which involves Fourier transform of external field coefficients, evaluation of transfer matrices at discrete frequencies, and their spline interpolation in the frequency domain, and finally inverse Fourier transform of the induced coefficients back to time domain 
with $1 \mathrm{hr}$ step, are given in Olsen and Kuvshinov (2004) and Kuvshinov and Olsen (2005).

Realistic Swarm satellite trajectories were simulated for the same period, the external and induced fields were synthetized along the tracks with $1 \mathrm{~Hz}$ sampling rate, together with other components of geomagnetic field (main, lithospheric, ionospheric and corresponding induced field), as well as a model of instrument noise. Detailed description on the test dataset assembly is given by Olsen et al. (2006). The CI was then used to reconstruct the individual field components, including the magnetospheric field and its induced counterpart (Sabaka et al., 2013).

Thus, the input for the inversion discussed here were 4.5 years long time series of reconstructed external and internal Gauss coefficients in the geomagnetic reference frame. Similarly to the checkerboard test, the external field was truncated at degree 3 and order 1, the internal field was modelled up to degree and order 5. The dipolar coefficients $g_{10}, q_{10}$ were provided with $1.5 \mathrm{hr}$ sampling, other coefficients were sampled at $6 \mathrm{hr}$ due to the limited longitudinal coverage of three satellites. Error estimates were also provided in the input dataset.

The coefficients were first preprocessed by rescaling to full normalization, using Eqs. (5)-(6). The non-dipolar coefficients were interpolated in time by cubic splines to common sampling interval of $1.5 \mathrm{hr}$. The conductivity model was parameterized using 5 layers each $200 \mathrm{~km}$ thick with spherical harmonic expansion truncated at $j_{\max }^{\rho}=5$. Note that the layer boundaries used in the inversion do not correspond to the layer boundaries of the target model. All computations were performed in the geomagnetic reference frame; however, the final conductivity model was rotated to the geographic coordinate system. The inversion was run using the $R_{1}$ regularization (21) for 8 values of $\lambda$, ranging from $10^{-5}$ to $10^{0}$. Based on an L-curve visual analysis (Fig. 3), we have chosen $\tilde{\lambda}=2 \times 10^{-2}$ as the optimal balance between data fit and model smoothness.

The resulting model is shown in the left panel of Fig. 2. The recovery of the uppermost $400 \mathrm{~km}$ is poor with spurious 3-D oscillations present below the oceans, and immediately above the large target heterogeneity. These can be assigned to combined effect of surface conductance, as observed in the checkerboard test, with the regularization term constraining the size of the conductivity jump across the $400 \mathrm{~km}$ boundary, and the lower sampling rate of nondipolar coefficients, reducing the information contained in the signal at periods below 1 day, which are the most sensitive to upper mantle conductivity. Obviously, given the limited lateral resolution of the data, it is difficult to resolve small scale features present in the upper $400 \mathrm{~km}$. However, the shape and conductivity of the large heterogeneity are well reconstructed in the $400-600 \mathrm{~km}$ depth range. In the 600-800 km span, the reconstructed model contains a combination of the heterogeneous layer in the target model with the underlying homogeneous, conductive layer; the conductivity below Pacific is well resolved, while the background value is increased elsewhere. Finally, the conductivity of the lower mantle is recovered as a 1-D structure, with small oscillations that are suppressed by regularization.

\section{Conclusions and Outlook}

We have developed and tested a new technique to invert a time series of Gauss coefficients representing the external and internal magnetic field in terms of a 3-D distribution of electrical conductivity, using direct integration of the magnetic field in the time domain. The approach is designed to process time series provided by the CI for the Swarm multisatellite mission (Olsen et al., 2013), and complements a traditional 3FDI approach (Püthe and Kuvshinov, 2013). We have demonstrated the ability to recover the shape and conductivity of a large heterogeneous sctructure positioned in the mid-mantle from a closed-loop simulation of the Swarm satellite mission that includes realistic model of satellite trajectories, instrument noise, and separation of signals of core, lithospheric, ionospheric, magnetospheric, and induced origin. Moreover, the induced signals for the test dataset were generated using an independent forward solver in the frequency-domain, introducing an additional source of discrepancies in forward modelling. The parameterization of the model space in the inverse problem did not conform to the target model, both in terms of layer interfaces positioned at different depths, and sharp lateral conductivity contrasts being fitted by spherical harmonics.

The time-domain method, and accompanying programs are currently being expanded. Use of full data covariance matrix, and external field adjustments taking into account its uncertainties are being investigated. Modifications of the regularization methods are also considered. In particular, since the electrical conductivity varies dominantly with depth, separation of the gradient or Laplacian operator in Eqs. (21)-(22) into the radial and angular parts, and downweighting the first one, could allow for larger radial variability without introducing spurious lateral oscillations to the results of the inverse problem.

Acknowledgments. The software chain described in this paper was developed under European Space Agency contract 4000102140/10/NL/1A, Development of the Swarm Level 2 Algorithms and Associated Level 2 Processing Facility. The support of the Grant Agency of the Czech Republic, project P210/11/1366, is also acknowledged. I thank M. E. Everett and A. Kuvshinov for their helpful comments.

\section{References}

Banks, R. J., Geomagnetic variations and the electrical conductivity of the upper mantle, Geophys. J. R. Astron. Soc., 17, 457-487, 1969.

Blackford, L. S., J. Choi, A. Cleary, E. D'Azevedo, J. Demmel, I. Dhillon, J. Dongarra, S. Hammarling, G. Henry, A. Petitet, K. Stanley, D. Walker, and R. C. Whaley, ScaLAPACK Users' Guide, Society for Industrial and Applied Mathematics, Philadelphia, 1997.

Dorn, O., H. Bertete-Aquirre, J. G. Berryman, and G. C. Papanicolaou, A nonlinear inversion method for 3D electromagnetic imaging using adjoint fields, Inverse Problems, 15, 1523-1558, 1999.

Everett, M. E., S. Constable, and C. G. Constable, Effects of near-surface conductance on global satellite induction responses, Geophys. J. Int., 153, 277-286, 2003.

Fichtner, A., Full Seismic Waveform Modelling and Inversion, SpringerVerlag, Berlin, Heidelberg, 2011.

Fornberg, B., A Practical Guide to Pseudospectral Methods, Cambridge Univ. Press, Cambridge, 1996.

Hamano, Y., A new time-domain approach for the electromagnetic induction problem in a three-dimensional heterogeneous earth, Geophys. J. Int., 150, 753-769, 2002.

Hansen, P., Analysis of discrete ill-posed problems by means of the Lcurve, SIAM Rev., 34, 561-580, 1992. 
Kelbert, A., G. D. Egbert, and A. Schultz, Non-linear conjugate gradient inversion for global EM induction: Resolution studies, Geophys. J. Int., 173(2), 365-381, 2008.

Kelbert, A., A. Schultz, and G. D. Egbert, Global electromagnetic induction constraints on transition-zone water content variations, Nature, $\mathbf{4 6 0}$, 1003-1007, 2009.

Koyama, T., H. Shimizu, H. Utada, M. Ichiki, E. Ohtani, and R. Hae, Water content in the mantle transition zone beneath the North Pacific derived from the electrical conductivity anomaly. in Earth's Deep Water Cycle, edited by S. Jacobsen and S. van der Lee, AGU Geophys. Monogr. Ser., 168, 171-179, 2006

Kuvshinov, A., 3-D Global induction in the oceans and solid Earth: Recent progress in modeling magnetic and electric fields from sources of magnetospheric, ionospheric and oceanic origin, Surv. Geophys., 29, 139_ 186, 2008.

Kuvshinov, A., Deep electromagnetic studies from land, sea and space: Progress status in the past 10 years, Surv. Geophys., 33, 169-209, 2012.

Kuvshinov, A. and N. Olsen, Modelling the ocean effect of geomagnetic storms at ground and satellite altitude, in Earth Observation with CHAMP, Results from Three Years Orbit, edited by Ch. Reigber, H. Lühr, P. Schwintzer, and J. Wickert, 353-358, Springer-Verlag, Berlin Heidelberg, 2005.

Kuvshinov, A. V. and N. Olsen, A global model of mantle conductivity derived from 5 years of CHAMP, Ørsted, and SAC-C magnetic data, Geophys. Res. Lett., 33, L18301, 2006.

Kuvshinov, A. and A. Semenov, Global 3-D imaging of mantle electrical conductivity based on inversion of observatory C-responses I. An approach and its verification, Geophys. J. Int., 189, 1335-1352, 2012.

Kuvshinov, A., J. Velímský, P. Tarits, A. Semenov, O. Pankratov, L. Tøffner-Clausen, Z. Martinec, N. Olsen, T. Sabaka, and A. Jackson, Level 2 products and performances for mantle studies with Swarm. Swarm Science Study under ESA contract, final report, 2010.

Lahiri, B. N. and A.T. Price, Electromagnetic induction in nonuniform conductors and the determination of the conductivity of the Earth from terrestrial magnetic variations, Phil. Trans. R. Soc. Lond., A237, 509$540,1939$.

Langel, R. A., Main field, in Geomagnetism, edited by J. A. Jacobs, 249512, Academic Press, London, 1987.

Martinec, Z. and J. Velímský, The adjoint sensitivity method of global electromagnetic induction for CHAMP magnetic data, Geophys. J. Int., 179, 1372-1396, 2009.

McGillivray, P. R., D. W. Oldenburg, R. G. Ellis, and T. M. Habashy, Calculation of sensitivities for the frequency-domain electromagnetic problem, Geophys. J. Int., 116(1), 1-4, 1994.

Olsen, N. and A. Kuvshinov, Modeling the ocean effect of geomagnetic storms, Earth Planets Space, 56, 525-530, 2004.

Olsen, N., F. Lowes, and T. Sabaka, Ionospheric and induced field leakage in geomagnetic field models, and derivation of candidate models for DGRF 1995 and DGRF 2000, Earth Planets Space, 57, 1191-1196,
2005

Olsen, N., R. Haagmans, T. J. Sabaka, A. Kuvshinov, S. Maus, M. E. Purucker, M. Rother, V. Lesur, and M. Mandea, The Swarm End-to-End mission simulator study: A demonstration of separating the various contributions to Earth's magnetic field using synthetic data, Earth Planets Space, 58(4), 359-370, 2006.

Olsen, N., E. Friis-Christensen, R. Floberghagen, P. Alken, C. D Beggan, A. Chulliat, E. Doornbos, J. T. da Encarnação, B. Hamilton, G. Hulot, J. van den IJssel, A. Kuvshinov, V. Lesur, H. Lühr, S. Macmillan, S. Maus, M. Noja, P. E. H. Olsen, J. Park, G. Plank, C. Püthe, J. Rauberg, P. Ritter, M. Rother, T. J. Sabaka, R. Schachtschneider, O. Sirol, C. Stolle, E. Thébault, A. W. P. Thomson, L. Tøffner-Clausen, J. Velímský, P. Vigneron, and P. N. Visser, The Swarm Satellite Constellation Application and Research Facility (SCARF) and Swarm data products, Earth Planets Space, 65, this issue, 1189-1200, 2013.

Pankratov, O., D. Avdeev, and A. Kuvshinov, Electromagnetic field scattering in a heterogeneous Earth: A solution to the forward problem, Phys. Solid Earth, 31, 201-209, 1995.

Press, W. H., S. A. Teukolsky, W. T. Vetterling and B. P. Flannery, Numerical Recipes in Fortran. The Art of Scientific Computing, Cambridge University Press, Cambridge, 1992.

Püthe, C. and A. Kuvshinov, Determination of the 3-D distribution of electrical conductivity in Earth's mantle from Swarm satellite data: Frequency domain approach based on inversion of induced coefficients, Earth Planets Space, 65, this issue, 1247-1256, 2013.

Sabaka, T. J., L. Tøffner-Clausen, and N. Olsen, Use of the Comprehensive Inversion method for Swarm satellite data analysis, Earth Planets Space, 65, this issue, 1201-1222, 2013.

Semenov, A. and A. Kuvshinov, Global 3-D imaging of mantle conductivity based on inversion of observatory C-responses C-responses. II, Data analysis and results, Geophys. J. Int., 191(3), 881-1470, 2012.

Shimizu, H., H. Utada, K. Baba, T. Koyama, M. Obayashi, and Y. Fukao, Three-dimensional imaging of electrical conductivity in the mantle transition zone beneath the North Pacific Ocean by a semi-global induction study, Phys. Earth Planet. Inter., 183, 262-269, 2010.

Tarits, P. and M. Mandea, The heterogeneous electrical conductivity structure of the lower mantle, Phys. Earth Planet. Inter., 183, 115-125, 2010.

Velímský, J. and C. C. Finlay, Effect of a metallic core on transient geomagnetic induction, Geochem. Geophys. Geosyst., 12, Q05011, 2011.

Velímský, J. and Z. Martinec, Time-domain, spherical harmonic-finite element approach to transient three-dimensional geomagnetic induction in a spherical heterogeneous Earth, Geophys. J. Int., 160, 81-101, 2005.

Velímský, J., Z. Martinec, and M. E. Everett, Electrical conductivity in the Earths mantle inferred from CHAMP satellite measurements, I. Data processing and 1-D inversion, Geophys. J. Int., 166, 529-542, 2006.

J. Velímský (e-mail: jakub.velimsky@mff.cuni.cz) 